\title{
Role of Microscopy as the Reference Method for Particle Size Analysis in Drug Development
}

\author{
L.M. DiMemmo, ${ }^{*}$ M. Hubert, B. Sarsfield, and B. Shekunov \\ * Bristol-Myers Squibb Co., One Squibb Drive, New Brunswick, NJ 08903
}

Characterizing size and shape of powder particles is critical for the development of a drug product since most pharmaceutical processes such as granulation, mixing and compaction are affected by these properties. An ideal particle characterization technique should provide an accurate determination of the volume-weighted particle size distribution (PSD) combined with a reliable definition of the particle shape. The majority of particle size techniques calculate particle size as a spherical equivalent. In addition, very few techniques directly measure particle shape. Each technique measures a different aspect of particle size to calculate an equivalent spherical diameter (ESD).

Several studies were initiated to determine the optimal techniques to characterize PSD and morphology and to develop correlations among techniques. The goal was to use optical microscopy with image analysis to confirm the accuracy of the other particle size techniques. Equivalent diameters from three particle size techniques including laser light scattering (LLS), focused beam reflectance (FBRM) and dynamic image analysis (DIA) were compared to optical microscopy with image analysis (OM/IA). Optical microscopy is considered the most accurate because the size and shape of the individual particles can be observed and measured [1]. Therefore, it can be used as a reference when comparing distributions from LLS, FBRM and DIA.

To do this comparison several factors were considered such as: relative size $(5 \mu, 50 \mu$ or $500 \mu)$ and shape (spherical or irregular) of the sample, objective and camera resolution [2], artifacts from image acquisition and data collection, sample size [3], spherical volume calculation and accuracy of the system based on analysis of a standard. The comparison of these techniques was simplified by first evaluating spherical beads (glass beads and polystyrene beads) since they have a single diameter and are within the size range for many active pharmaceutical ingredients (APIs). The study was extended to look at irregular shaped particles using a common pharmaceutical excipient Avicel ${ }^{\circledR}$ PH-101 microcrystalline cellulose (MCC).

Accuracy was confirmed for spherical and irregular shaped particles and is shown in Figure 1 and Figure 2. Knowledge of OM/IA artifacts was developed. OM/IA is the optimum technique to analyze standards, but artifacts may influence the results of real samples as shown in Figure 3 and Figure 4. Details of volume calculations, sample size optimization and instrumental artifacts related to $\mathrm{OM} / \mathrm{IA}$ will be presented [4].

References

[1] B. Y. Shekunov et al., Pharmaceutical Research 24:2 (2007) 203-227.

[2] Particle size analysis-image analysis methods-Part 1: Static image analysis methods, ISO 133221:2004.

[3] Particulate materials-Sampling and sample splitting for the determination of particulate properties, ISO 14488:2007. 
[4] Students S. DeLeon, A. Patel, V. Rosa, of the Drexel University Co-op Program are gratefully acknowledged for their help with data acquisition.
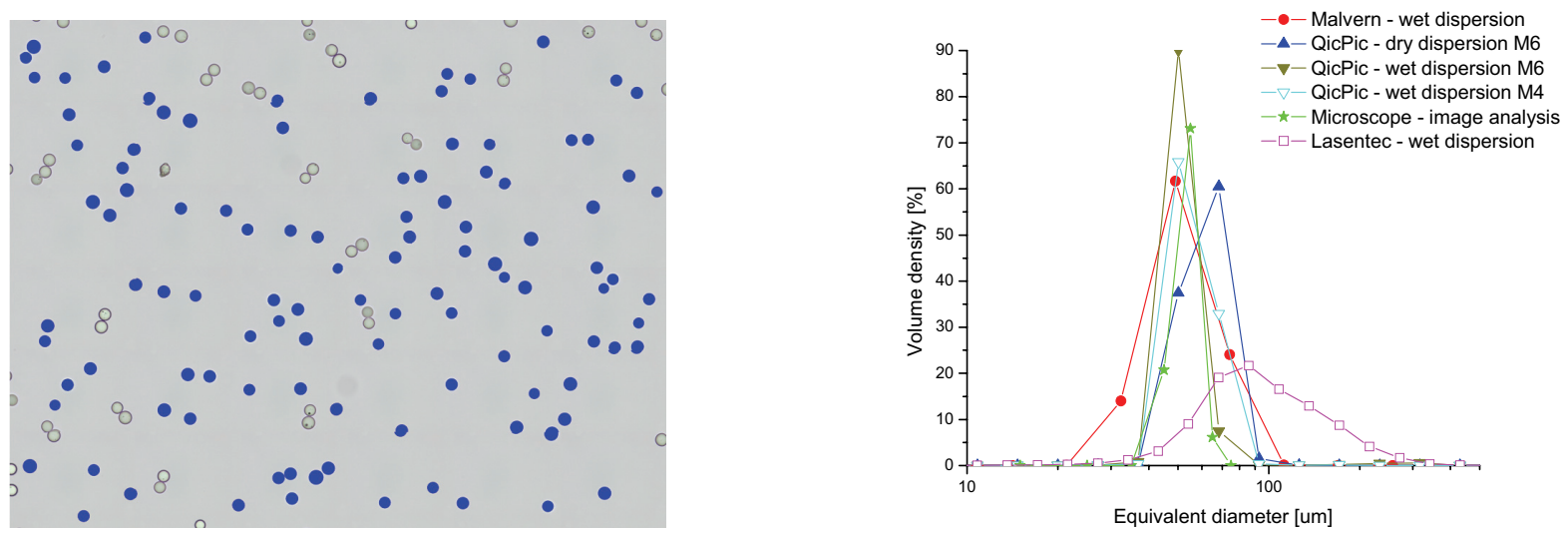

Figure 1. Optical microscopy binary image of mosaic for polystyrene beads DRI-CAL $®$ DC-50 (left) and overlay of spherical volume distribution for OM/IA, DIA, LLS and FBRM. PSD by LLS (wet method) and DIA (wet method) is similar to OM/IA. DIA (dry) and FBRM results were larger.
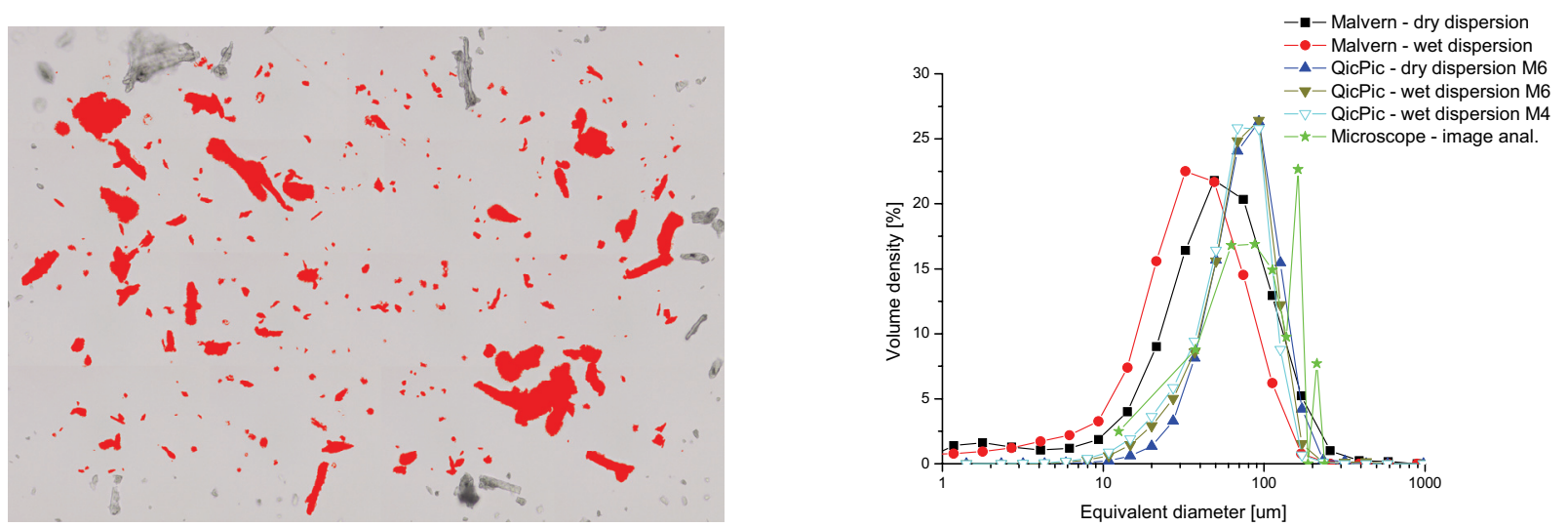

Figure 2. Optical microscopy binary image of mosaic for pharmaceutical excipient Avicel ${ }^{\circledR}$ PH-101 MCC (left) and overlay of spherical volume distribution for OM/IA, DIA and LLS. PSD by LLS (wet and dry methods) and DIA (wet and dry methods) is different than OM/IA. OM/IA results were larger.

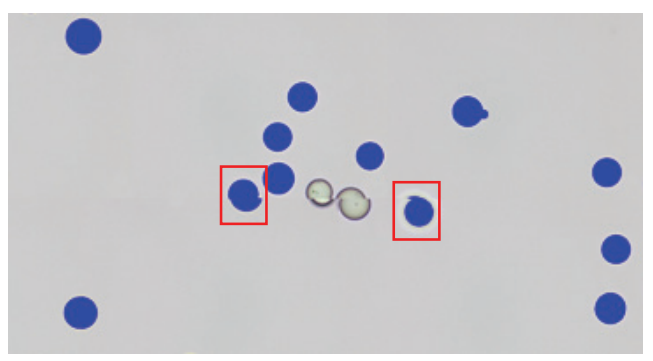

Figure 3. Mis-aligned particle images affect sphericity results. Particles framed in red showed low sphericity.

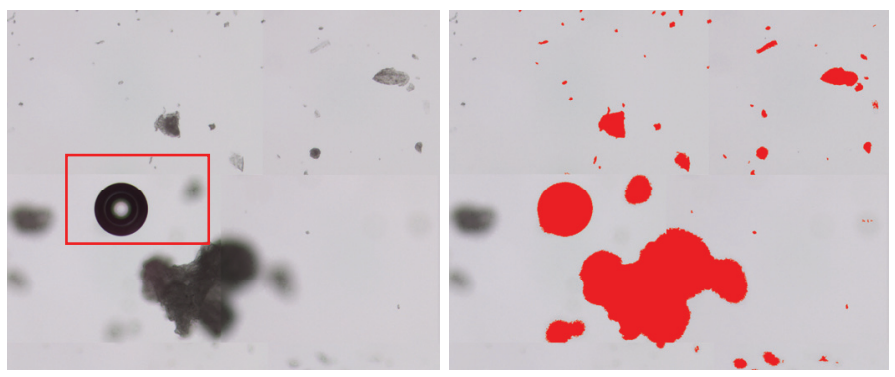

Figure 4. Sometimes particles out of focus or air bubbles are measured. 